\title{
From Bouncing to Floating: The Leidenfrost Effect with Hydrogel Spheres
}

\author{
Scott Waitukaitis, ${ }^{1,2}$ Kirsten Harth, ${ }^{3}$ and Martin van Hecke ${ }^{1,2}$ \\ ${ }^{1}$ AMOLF, Science Park 104, 1098 XG Amsterdam, Netherlands \\ ${ }^{2}$ Huygens-Kamerlingh Onnes Laboratory, Universiteit Leiden, P.O. Box 9504, 2300 RA Leiden, Netherlands \\ ${ }^{3}$ Physics of Fluids Group, Mesa+Institute, University of Twente, 7500 AE Enschede, Netherlands
}

(Received 26 January 2018; revised manuscript received 6 June 2018; published 25 July 2018)

\begin{abstract}
The Leidenfrost effect occurs when a liquid or stiff sublimable solid near a hot surface creates enough vapor beneath it to lift itself up and float. In contrast, vaporizable soft solids, e.g., hydrogels, have been shown to exhibit persistent bouncing-the elastic Leidenfrost effect. By carefully lowering hydrogel spheres towards a hot surface, we discover that they are also capable of floating. The bounce-to-float transition is controlled by the approach velocity and temperature, analogously to the "dynamic Leidenfrost effect." For the floating regime, we measure power-law scalings for the gap geometry, which we explain with a model that couples the vaporization rate to the spherical shape. Our results reveal that hydrogels are a promising pathway for controlling floating Leidenfrost objects through shape.
\end{abstract}

DOI: 10.1103/PhysRevLett.121.048001

It is a common observation during cooking that droplets of water on a pan whose temperature far exceeds $100^{\circ} \mathrm{C}$ do not boil, but instead gently glide around the surface. This is the archetypal manifestation of the Leidenfrost effect, which occurs due to the development of a vapor layer that supports the weight of the droplet and prevents it from entering into contact boiling [1-15]. The same floating is also possible with sublimable stiff solids such as dry ice [16-19], and in both cases the physics is governed by an interplay of vaporization, squeeze flow, and force balance [6]. These dynamics are markedly different from the elastic Leidenfrost effect, which occurs with vaporizable soft solids [20,21]. Rather than floating, water-saturated hydrogel spheres ( $\sim 99 \%$ water by mass) dropped onto hot surfaces exhibit sustained bouncing (lasting up to several minutes) accompanied by shrill noise generation. The physics in this situation arises from a coupling between vaporization and the gel's elastic deformations, which lead to high-frequency, energy-harvesting oscillations in pressure and deformation at the interface during each impact that both produce the sound and keep the object bouncing.

Fundamental questions regarding the floating and bouncing Leidenfrost effects remain unresolved. Is it possible for vaporizable soft solids to float rather than bounce, and if so how does one access the floating regime? What triggers this transition? How would the floating of a polymer-laden gel compare to that which occurs with pure liquids or solids? In this Letter, we answer these questions through a series of experiments that connect the floating and bouncing Leidenfrost phenomena and probe the slow evolution of the floating state. First, we show that vaporizable soft solids can exhibit Leidenfrost floating, provided they approach the hot surface at sufficiently small velocities. Second, the energy-harvesting oscillations (which cause the sustained bouncing and screeching in the elastic Leidenfrost effect) are only triggered if the gel physically touches the surface. Third, we show that the polymer matrix remains intact as the water vaporizes, but does not hinder the floating mechanism until the latest stages. This last observation in particular allows us to develop a model that couples the gel's shape to its vaporization rate and predict the time-dependent floating behavior. Given hydrogels are easily polymerized and cast into complex forms, our results offer a new and robust pathway for precisely controlling the shape evolution and dynamics of Leidenfrost-levitated objects.

Bounce-to-float transition.-The images in Figs. 1(a) and 1(b) show the characteristic behaviors for the bouncing and floating Leidenfrost regimes (for videos, see Supplemental Material [22]). We lower swollen, polyacrylamide hydrogel spheres ( $\sim 99 \% \mathrm{H}_{2} \mathrm{O}$ by mass) at constant velocity $v_{0}$ toward a stainless steel surface at a temperature $T$ [experimental setup in Fig. 1(c)]. Unless otherwise indicated, the spheres have masses in the range $M=$ $1.36 \pm 0.08 \mathrm{~g}$ and radii $R=6.9 \pm 0.1 \mathrm{~mm}$. For a "fast" velocity of $1.6 \times 10^{-1} \mathrm{~m} / \mathrm{s}$, the sphere exhibits sustained bouncing behavior that is amplified over time, i.e., the elastic Leidenfrost effect. In contrast, for a much smaller approach velocity $\left(v_{0}=1.5 \times 10^{-4} \mathrm{~m} / \mathrm{s}\right)$, the sphere gently glides across the surface, strongly suggesting that it has entered a floating Leidenfrost state.

To unambiguously determine if such a sphere touches the surface, we use an electrocontact technique. A thin copper wire [visible in Figs. 1(a) and 1(b)] connects the sphere to a dc voltage supply with output $V=10 \mathrm{~V}$. The hot surface is connected to ground via a resistor $\left(R_{p}=4.6 \mathrm{~K} \Omega\right)$, and by measuring the voltage drop across this resistor we probe for contact. If the sphere floats and 
(a)

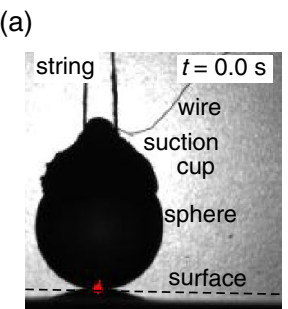

(b)

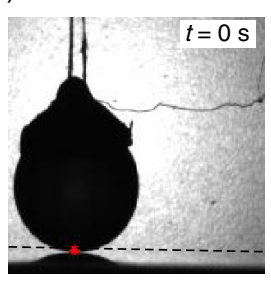
$v_{O}=1.6 \times 10^{-1} \mathrm{~m} / \mathrm{s}$
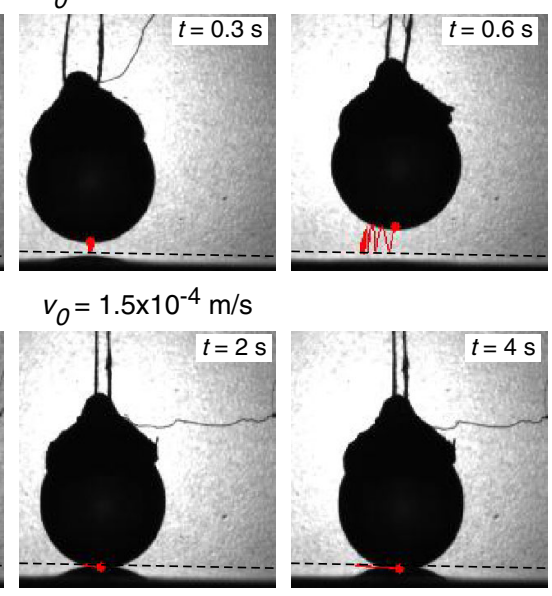

(c)

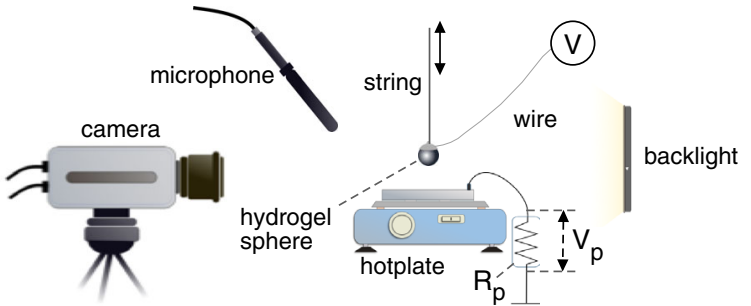

(d)
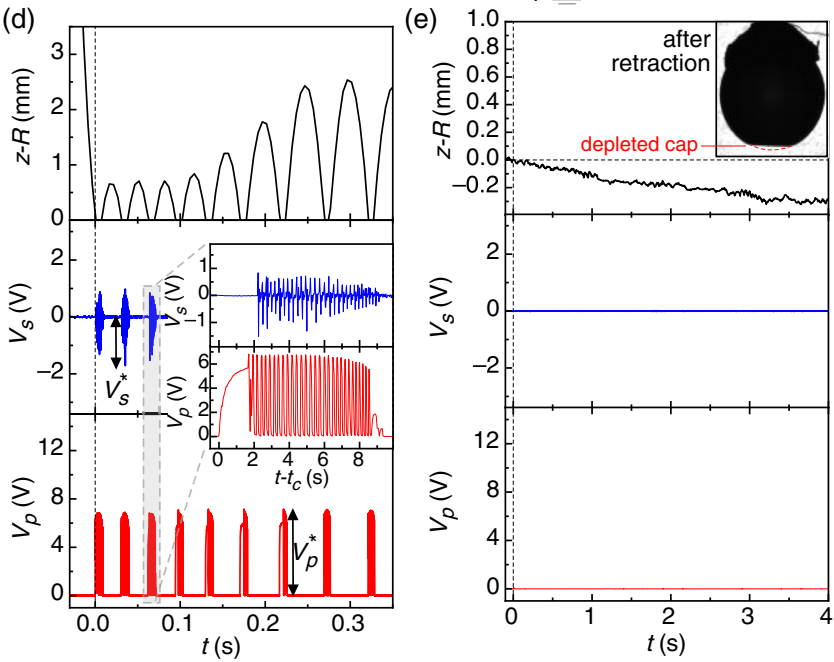

FIG. 1. (a) A hydrogel sphere is lowered toward a $T=215^{\circ} \mathrm{C}$ stainless steel surface at an approach velocity, $v_{0}=1.6 \times 10^{-1} \mathrm{~m} / \mathrm{s}$. The bounce height of the sphere (red trace) grows with time (video in the Supplemental Material [22]). (b) For a much slower velocity $\left(v_{0}=1.5 \times 10^{-4} \mathrm{~m} / \mathrm{s}\right)$, the sphere glides along the surface. (c) Surface contact is measured by connecting the sphere to a voltage source $V$ and measuring the voltage drop across the resistor $R_{p}$. (d) Center of mass vertical position $z-R$ (top), audio signal (middle), and plate voltage (bottom) for panel (a); the inset shows the rapid oscillations in the sound and contact during a single "impact." (e) Same measurements for the run of panel (b) show no sound or contact; the inset shows that a spherical cap of height $\sim 300 \mu \mathrm{m}$ is depleted after retraction.

makes no contact, the voltage across this resistor remains near to its noise value, but if the sphere touches the surface then the voltage jumps to a value close to $10 \mathrm{~V}$ [Fig. 1(c); see Supplemental Material [22] for details]. Concurrently, we listen with a microphone to detect screeching and sync all measurements. Figure 1(d) shows the center of mass position $(z-R$, i.e., relative to when the sphere should just make contact), audio signal, and plate voltage for the fast approach. In addition to confirming the growing bounce height and noise generation that are characteristic of the elastic Leidenfrost effect [20], oscillations are visible in the plate voltage, which shows that the sphere bottom rapidly makes and breaks physical contact with the surface during each impact [inset to Fig. 1(d); for a full description of these oscillations, we refer the reader to [20] ]. In Fig. 1(e), we plot the same signals for the slow approach, which highlight two key distinctions. First, there is no physical contact. Second, at longer timescales, the sphere appears to sink into the surface as $z-R<0$. Upon retraction, we see that this is caused by the depletion of a small spherical cap on the bottom of the sphere [inset photo in Fig. 1(e)].

In order to determine where the transition between floating and bouncing occurs, we probe a range of velocities spanning between the extremes of Fig. 1. Because of the occasional detection of spurious contacts (occurring in $\sim 15 \%$ of the floating runs), we perform ten runs at each velocity to produce a statistically significant sample (see Supplemental Material [22] for details on the measurement variability). In each run, we measure the maximum plate voltage $V_{p}^{*}$ and maximum (absolute) audio voltage $V_{s}^{*}$ [indicated in Fig. 1(d)]. In Figs. 2(a) and 2(b), we plot the median values over the ten runs of $V_{p}^{*}$ and $V_{s}^{*}$ at each $v_{0}$, as well as the individual points from each run. The median values exhibit a clear transition at $v_{T}=4.3 \pm 0.5 \mathrm{~mm} / \mathrm{s}$, before which virtually all runs exhibit no contact and no sound and after which all exhibit both contact and sound.

To study this transition in more detail, we probe the observed behavior at several different temperatures [Fig. 2(c); for all data see the Supplemental Material [22]]. We find that the transition velocity grows approximately linearly with the plate temperature at a rate of $70 \pm 10 \mu \mathrm{m} /(\mathrm{sK})$. This linear trend predicts a threshold temperature of $T_{c}=160 \pm 4{ }^{\circ} \mathrm{C}$. The existence of this threshold for floating is supported by the fact that, down the lowest velocities we can reliably probe $(\sim 100 \mu \mathrm{m} / \mathrm{s})$, we observe no floating for a plate temperature of $155^{\circ} \mathrm{C}$. These data warrant comparison with the dynamic Leidenfrost transition observed for liquid droplets impacting a heated substrate, where a similar separation between contact and noncontact plays out as a function of impact speed and temperature [4,5,23]. Physically, one suspects that with the extremely low velocities we probe here, the mechanism is dependent upon whether or not sufficient viscous stress can build up in the vapor layer to prevent the sphere from touching - similar to what happens for liquids impacting at low velocities [23]. Important distinctions exist, however, that preclude exact comparison. We observe transition speeds on the order of millimeters 

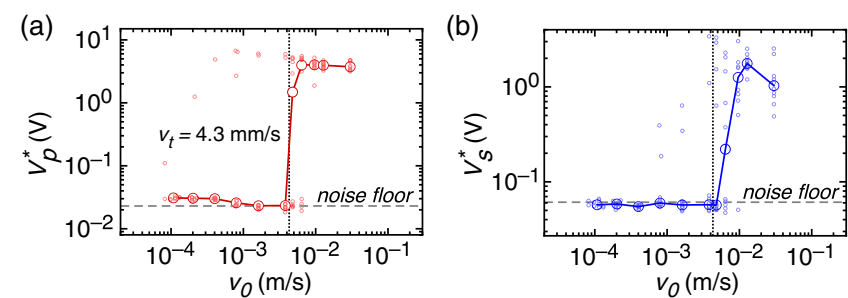

(c)

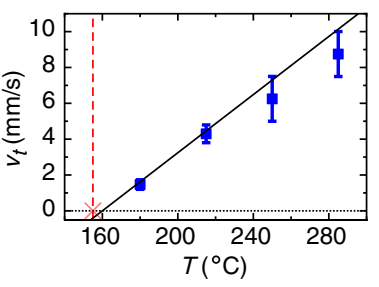

(d)

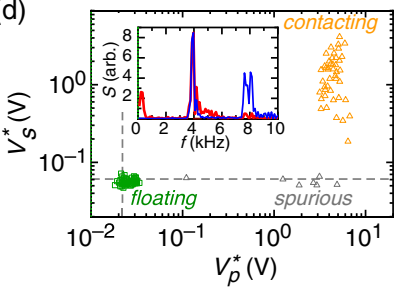

FIG. 2. (a) Maximum plate voltages $V_{p}^{*}$ vs approach velocity $v_{0}$ for spheres approaching a plate at $215^{\circ} \mathrm{C}$. At each $v_{0}$ we do 10 runs and plot the individual data points (small red circles) and median value (large red circles and line). A transition from noncontact to contact is present at $v_{T}=4.3 \pm 0.5 \mathrm{~mm} / \mathrm{s}$. (b) Same as (a) but using the maximum (absolute) value of the audio signal $V_{s}^{*}$. (c) The transition velocity grows approximately linearly with temperature and floating is only possible for temperatures greater than the threshold $T_{c}=160 \pm 4{ }^{\circ} \mathrm{C}$ (see Supplemental Material [22] for full data sets for all temperatures). (d). Scatter plot of all $V_{s}^{*}$ vs $V_{p}^{*}$ (again at $215^{\circ} \mathrm{C}$ ) with 110 runs total; 58 floating runs (green squares), 42 contacting runs (orange triangles), and 10 spurious contact runs (gray triangles). Inset: power spectra of the audio (blue) and plate voltages (red) for the single impact shown in the inset of Fig. 1(d).

per second, whereas for liquid droplets at similar temperatures the transition typically occurs at meters per second. This difference must be due in large part to the vastly larger size of our spheres, which are 1000 times more massive. A second important distinction is that the deformations in the liquid case are managed by surface tension (and hence characterized by the dimensionless Weber number), whereas in our case elasticity plays a dominant role. We leave fully investigating this transition with elasticity as the dominant factor as a subject of future work.

By scrutinizing the voltage and sound data further, we gain an additional insight about the elastic Leidenfrost effect-the energy-harvesting oscillations responsible for sustained bouncing are triggered by physical contact. This can be seen by taking all data and plotting $V_{s}^{*}$ vs $V_{p}^{*}$ [Fig. 2(d)], which reveals that contact can occur independently of sound generation (spurious contacts caused by flakes of gel peeling off and grazing the surface-see Supplemental Material [22]), but not vice versa. This is further evidenced by comparing the power spectra for the audio and plate voltages, which both share the same fundamental peak $[\sim 4 \mathrm{kHz}$ in the inset to Fig. 2(d)]. Interestingly, similar oscillations have been observed for liquids $[5,23]$, but in those experiments no energy harvesting is observed and instead droplets are quickly destroyed by the vigorous contact boiling.

The floating regime.-We now turn our attention exclusively to the floating regime, where we (1) identify the evolution of a vapor gap below the sphere, (2) elucidate the role of the polymer matrix, and (3) reveal that the spherical shape of the gel has a direct impact on the time-dependent gap geometry. We use the modified setup shown in Fig. 3(a), where we tilt the hotplate at an angle $\theta \approx 45^{\circ}$ and lower the spheres until the string makes an angle $\phi \approx 10^{\circ}$ with the vertical. This setup prevents the sphere from gliding out of view and reduces the effect of gravity. Lowering the sphere with a force sensor (Instron 5965), we simultaneously measure the tension necessary to maintain its position. Given mechanical equilibrium, this gives us the unique ability to directly calculate the lift force provided by the vapor and correlate it to the gap development (see Supplemental Material [22]). Given that (1) spheres undergo mechanical rupture after $3-4 \mathrm{~min}$ (for video of rupture, see Supplemental Material [22]) and (2) exit our field of view after $\sim 2 \mathrm{~min}$, we restrict our study here to this early regime and save probing longer times for future work.

In Fig. 3(b) we show images of a floating sphere (for video, see Supplemental Material [22]). At early times, no gap is visible within the resolution of our setup $(\sim 20 \mu \mathrm{m})$, but after about $5 \mathrm{~s}$ a clearly visible gas layer develops that isolates the sphere from the hot surface. Simultaneously, we observe that the bottom of the sphere is progressively widened and flattened-thus explaining the truncation in the inset of Fig. 1(e). What happens to the polymer matrix during these shape changes? We observe that after retraction, the bottom face appears noticeably dryer [Fig. 3(c) and video in the Supplemental Material [22]]. As can be seen in the video data, this patch has a finite thickness, yielding a subtle "bump" on the center of the flattened face that grows toward the hot surface. If we suspend a truncated sphere in the ambient environment, it regains its spherical shape and wet luster on a timescale of a few hours [Fig. 3(d)]. This reveals that the matte texture of the patch immediately after an experiment is due to the development of a dry polymer "skin," which remains intact and reusable.

One might expect that the presence of this growing skin could hinder the floating mechanism by making it more difficult for vapor to escape. To test for this, we make a quantitative comparison between our data and what occurs for a floating liquid or stiff solid. In those cases [14,18], it can be shown that the gap height is related to the radius and vapor force by

$$
h=\left(\frac{3 \pi \eta \kappa \Delta T}{2 \rho L}\right)^{1 / 4} \frac{r}{F^{1 / 4}}
$$

where $\eta=2 \times 10^{-5} \mathrm{Pas}, \quad \kappa=3 \times 10^{-2} \mathrm{~W} / \mathrm{m} \mathrm{K}, \quad \rho=$ $5 \times 10^{-1} \mathrm{~kg} / \mathrm{m}^{3}$, and $\Delta T=115^{\circ} \mathrm{C}$ are the vapor viscosity, thermal conductivity, density, and the temperature difference between the surface and the sphere (respectively), and $L$ is the latent heat. Any increase in the resistance to 
(a)

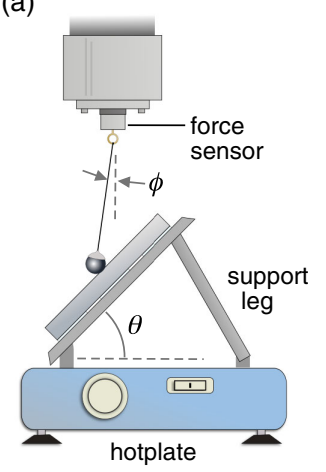

(b)

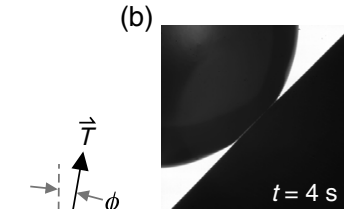

(d)

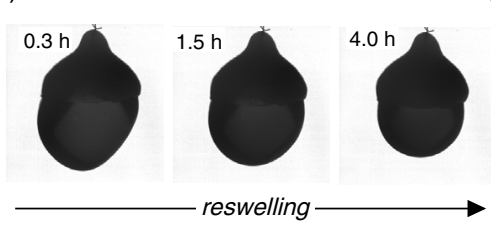

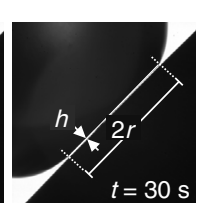

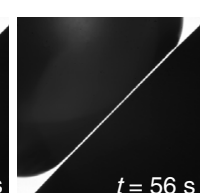

(e) 4.0
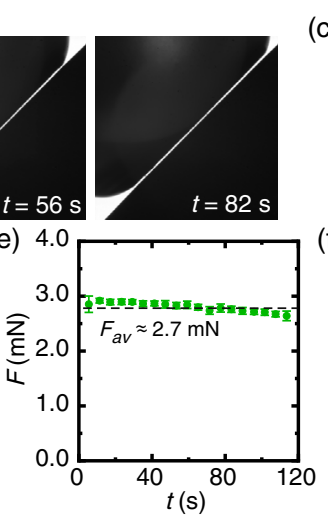

(c)
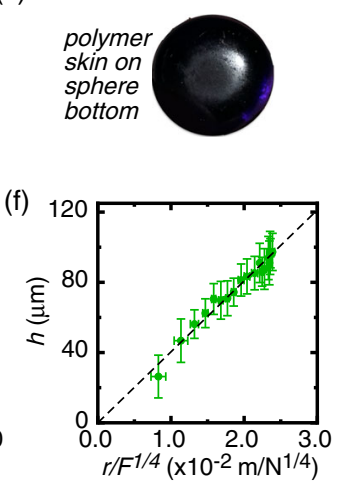

FIG. 3. (a) Modified setup where we use a slanted plate $\left(\theta \approx 45^{\circ}\right.$ and $\left.\phi \approx 10^{\circ}\right)$ and measure the tension in the string with a force sensor. (b) Images after time $t$ since the sphere first touches the surface reveal a visible gap whose height and radius increase with time. (c) Dried out bottom of the sphere after an experiment. (d) The truncated bottom of a sphere reswells on a timescale of a few hours. (e) The lift force provided by the vapor is nearly constant. (f) Plot of the gap height $h$ vs the parameter $r / F^{1 / 4}$, which when fitted to Eq. (1) corresponds to a latent heat, $L=(2.6 \pm 0.4) \times 10^{6} \mathrm{~J} / \mathrm{kg}$.

escaping vapor should appear as a change in $L$, which we therefore consider a free parameter. Using the images of the gap and the measurements from the force sensor [Fig. 3(e)], we directly measure all of these quantities and in Fig. 3(f) test them against this model. The data reveal that $h$ is indeed proportional to $r / F^{1 / 4}$ for the measurement times we are able to access. Fitting for $L$, we find a value of $(2.6 \pm 0.4) \times 10^{6} \mathrm{~J} / \mathrm{kg}$ - on par for normal water $\left(2.23 \times 10^{6} \mathrm{~J} / \mathrm{kg}\right)$. In the Supplemental Material, we confirm that these trends persist for spheres whose masses vary by an order of magnitude and for temperatures ranging from $180-300{ }^{\circ} \mathrm{C}$. The agreement of our data with Eq. (1) leads us to conclude that the vaporization and floating are largely unaffected by the growing polymer skin, which is consistent with recent experiments involving freely drying hydrogels that showed that the evaporative flux is essentially constant throughout the entire drying process [24].

Having clarified the role of the polymer, we now develop a model to predict how the gap evolves over time by considering the coupling between the gel's overall shape and the rate at which it vaporizes. At a given instant, the quantity of water liberated corresponds to the spherical cap with mass, $M_{\text {cap }}$. In the limit where the volume of this cap is much smaller than the sphere volume, one can approximate

$$
M_{\text {cap }} \approx \frac{\pi \rho_{g} r^{4}}{4 R},
$$

where $\rho_{g}=10^{3} \mathrm{~kg} / \mathrm{m}^{3}$ is the density of the gel. The rate at which this cap disappears corresponds to the thermally driven evaporative loss via conduction through the vapor layer, which is given by

$$
\dot{M}_{\text {cap }}=\frac{\kappa \Delta T \pi r^{2}}{L h} \text {. }
$$

Differentiating Eq. (2) and setting it equal to Eq. (3), one arrives at the differential equation

$$
\dot{r}=\frac{\kappa \Delta T R}{L h \rho_{g} r} .
$$

As in Eq. (1), $h \propto r / F^{1 / 4}$, and given that Fig. 3(e) shows that $F$ is essentially constant, we can approximate $h \propto r$ and solve the above equation to conclude $r=C_{1} t^{1 / 3}$ and $h=C_{2} t^{1 / 3}$ (see Supplemental Material for exact derivation and coefficients [22]). In Fig. 4 we plot the measured values for $r(t)$ and $h(t)$, which shows that they are consistent with this power-law scaling (and with appropriate fit values for $L$ ). In the Supplemental Material, we perform additional experiments changing both the sphere size and plate temperature and collapse all data for the time evolution of $h$ and $r$ onto two parameter-free master curves, thus fully validating our model. The consistency of our data with this model means that, for the parameters we can access, the Young's modulus of the gel plays no role-the floating evolution is determined entirely by the thermally driven vaporization rate and the spherical shape.

Implications and outlook.-Our results bear a number of implications for future work. First, while our data on the
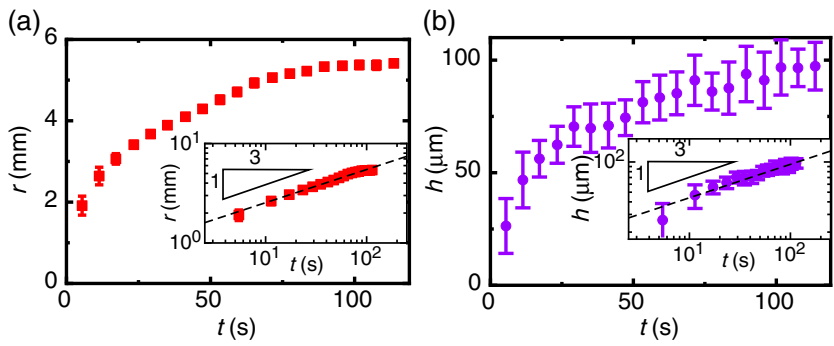

FIG. 4. Plots of the gap radius (a) and height (b) vs time. The insets show that $r$ and $h$ grow with power laws consistent with $t^{1 / 3}$. Fitting to these parameters for $L$, we find values of $(2.5 \pm 0.1) \times 10^{6} \mathrm{~J} / \mathrm{kg}$ and $(2.31 \pm 0.02) \times 10^{6} \mathrm{~J} / \mathrm{kg}$, respectively (see Supplemental Material for details [22]). 
transition from noncontact and floating at low velocities to contact and sustained bouncing at high velocities is remarkably similar to the dynamic Leidenfrost transition observed for liquids, much work remains to be done to sort out how these two situations compare. In particular, while the liquid transition is governed by the Weber number, it is not clear what controls the transition in the case of the solid hydrogels. Regarding the floating we are able to look at timescales ranging from a few to $\sim 100 \mathrm{sec}$, but we expect different behaviors at both earlier and later times. For earlier times, one would anticipate that the softness of the gel should play a role, thus providing a cutoff to the $t^{1 / 3}$ power laws we observe here. Extrapolating our $r(t)$ fit to find when it becomes smaller than the Hertzian contact radius of a sphere under its own weight $(\sim 1 \mathrm{~mm})$, we expect to see such effects for times less than $\sim 1 \mathrm{~s}$ and gap heights less than $\sim 10 \mu \mathrm{m}$. For larger times, we expect that the growing polymer skin will play a role by contacting the surface and/or providing a resistive tension to the lower face [25]. Nonetheless, our results show that such effects can be ignored for as long as a couple of minutes. Finally, while controlled motion of floating liquids or stiff solids has been achieved by using ratcheted surfaces or other asymmetries $[9,11,13,16,18,19]$, with hydrogels such mechanisms can be embedded into the object itself. Our results therefore open the door to functionalizing floating Leidenfrost objects through shape.

We thank A. Zuiderwijk for productive discussions. J. Mesman provided valuable technical support. We acknowledge funding from the Netherlands Organization for Scientific Research through Grants VICI No. NWO680-47-609 (M. v. H. and S. W.) and VENI No. NWO-68047-453 (S. W.), and from the German Science Foundation through Grant No. HA8467/1-1 (K. H.).

[1] S. Hidalgo-Caballero, Y. Escobar-Ortega, and F. PachecoVázquez, Phys. Rev. Fluids 1, 051902 (2016).

[2] J. H. Snoeijer, P. Brunet, and J. Eggers, Phys. Rev. E 79, 036307 (2009).

[3] F. Celestini, T. Frisch, and Y. Pomeau, Phys. Rev. Lett. 109, 034501 (2012).
[4] T. Tran, H. J. J. Staat, A. Prosperetti, C. Sun, and D. Lohse, Phys. Rev. Lett. 108, 036101 (2012).

[5] M. Shirota, M. A. J. van Limbeek, C. Sun, A. Prosperetti, and D. Lohse, Phys. Rev. Lett. 116, 064501 (2016).

[6] D. Quéré, Annu. Rev. Fluid Mech. 45, 197 (2013).

[7] X. Ma, J.-J. Liétor-Santos, and J. C. Burton, Phys. Fluids 27, 091109 (2015).

[8] J. C. Burton, A. L. Sharpe, R. C. A. van der Veen, A. Franco, and S. R. Nagel, Phys. Rev. Lett. 109, 074301 (2012).

[9] T. R. Cousins, R. E. Goldstein, J. W. Jaworski, and A. I. Pesci, J. Fluid Mech. 696, 215 (2012).

[10] V. Bertola, Int. J. Heat Mass Transfer 52, 1786 (2009).

[11] G. Lagubeau, M. Le Merrer, C. Clanet, and D. Quéré, Nat. Phys. 7, 395 (2011).

[12] I. U. Vakarelski, N. A. Patankar, J. O. Marston, D. Y. C. Chan, and S. T. Thoroddsen, Nature (London) 489, 274 (2012).

[13] H. Linke, B. J. Alemán, L. D. Melling, M. J. Taormina, M. J. Francis, C. C. Dow-Hygelund, V. Narayanan, R. P. Taylor, and A. Stout, Phys. Rev. Lett. 96, 154502 (2006).

[14] A.-L. Biance, C. Clanet, and D. Quéré, Phys. Fluids 15, 1632 (2003).

[15] S. Dorbolo, N. Vandewalle, and B. Darbois Texier, Phys. Fluids 28, 123601 (2016).

[16] G. G. Wells, R. Ledesma-Aguilar, G. McHale, and K. Sefiane, Nat. Commun. 6, 6390 (2015).

[17] J. C. Burton, P. Y. Lu, and S. R. Nagel, Phys. Rev. E 88, 062204 (2013).

[18] G. Dupeux, T. Baier, V. Bacot, S. Hardt, C. Clanet, and D. Quéré, Phys. Fluids 25, 051704 (2013).

[19] T. Baier, G. Dupeux, S. Herbert, S. Hardt, and D. Quéré, Phys. Rev. E 87, 021001(R) (2013).

[20] S. R. Waitukaitis, A. Zuiderwijk, A. Souslov, C. Coulais, and M. van Hecke, Nat. Phys. 13, 1095 (2017).

[21] J. T. Pham, M. Paven, S. Wooh, T. Kajiya, H.-J. Butt, and D. Vollmer, Nat. Commun. 8, 905 (2017).

[22] See the Supplemental Material at http://link.aps.org/ supplemental/10.1103/PhysRevLett.121.048001 for videos of the experiments and details regarding the calculations and measurement techniques.

[23] M. Khavari and T. Tran, Phys. Rev. E 96, 043102 (2017).

[24] T. Bertrand, J. Peixinho, S. Mukhopadhyay, and C. W. MacMinn, Phys. Rev. Applied 6, 064010 (2016).

[25] J. B. Brown, Am. J. Phys. 31, 397 (1963). 